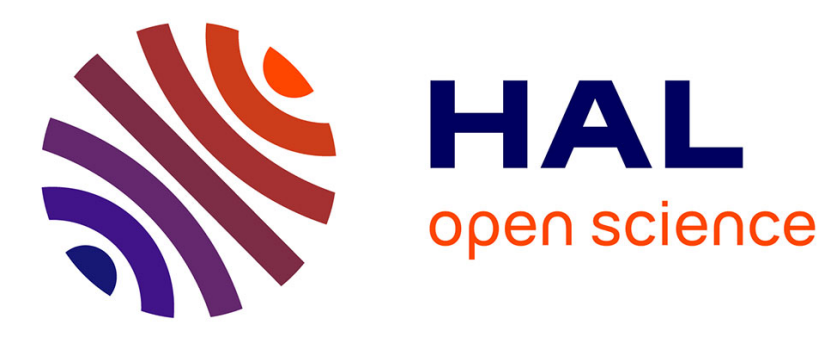

\title{
Inverse Problem Approach to Characterize and Model Magnetization Changes in a Thin Shell Structure Undergoing Magneto-Mechanical Effects
} Antoine Viana, Laure-Line Rouve, Olivier Chadebec, Gilles Cauffet, Jean-Louis Coulomb

\section{To cite this version:}

Antoine Viana, Laure-Line Rouve, Olivier Chadebec, Gilles Cauffet, Jean-Louis Coulomb. Inverse Problem Approach to Characterize and Model Magnetization Changes in a Thin Shell Structure Undergoing Magneto-Mechanical Effects. IEEE Transactions on Magnetics, 2011, 47 (5), pp.1450 1453. 10.1109/TMAG.2010.2097242 . hal-00595031

\section{HAL Id: hal-00595031 \\ https://hal.science/hal-00595031}

Submitted on 23 May 2011

HAL is a multi-disciplinary open access archive for the deposit and dissemination of scientific research documents, whether they are published or not. The documents may come from teaching and research institutions in France or abroad, or from public or private research centers.
L'archive ouverte pluridisciplinaire $\mathbf{H A L}$, est destinée au dépôt et à la diffusion de documents scientifiques de niveau recherche, publiés ou non, émanant des établissements d'enseignement et de recherche français ou étrangers, des laboratoires publics ou privés. 


\title{
Inverse Problem Approach to Characterize and Model Magnetization Changes in a Thin Shell Structure Undergoing Magneto-Mechanical Effects
}

\author{
A. Viana, L.-L. Rouve, O. Chadebec, G. Cauffet, and J.-L. Coulomb \\ Grenoble Electrical Engineering Lab, Université de Grenoble (Grenoble-INP, UJF, CNRS UMR 5269), \\ 38402 Saint Martin d'Hères, France
}

\begin{abstract}
Direct measurement of magnetization $\mathrm{M}$ inside a complex ferromagnetic geometry is generally impossible. In this paper, we propose to use an inverse problem algorithm to determine the law of variation of $M$ for such structures, accounting for the magneto-mechanical effects it is undergoing. The analytical law obtained leads to an intrinsic characterization model of magnetostriction inside the structure. Validation of the approach is achieved on a prototype undergoing high mechanical stresses in low magnetic field, by comparison with the predicted magnetic signature in the vicinity of the prototype, and measurements performed on external magnetic sensors.
\end{abstract}

Index Terms-Inverse problem, Jiles, magneto-mechanical.

\section{ORIGINALITY OF THE STUDY}

$\mathbf{T}$ $\mathrm{O}$ anticipate magnetization variations due to stress, some models can be found in literature but applications generally deal with simple shapes, free of demagnetizing field effects, such as rods, undergoing low mechanical stress levels, in a high magnetic field. In addition, the main mechanical stress is usually applied in the same direction as the field.

In our application (a ferromagnetic cylinder internally pressurized), high mechanical stresses are applied to a thin ferromagnetic geometry under low magnetic fields (less than $50 \mu \mathrm{T}$ ) in any direction. In this case, because of demagnetizing field, magnetization $\mathbf{M}$ is no longer homogeneous and cannot be simply measured. For this reason, to characterize magnetostriction, our interest has been first focused on a global feature: the external magnetic induction $\mathbf{B}$ measured by magnetic sensors outside the ferromagnetic body. It was previously shown [1] that, for thin shells, the law of evolution for external $\mathbf{B}$ was necessarily the same as for $\mathbf{M}$. This result was validated on a ferromagnetic cylinder undergoing an internal increasing pressure. An analytical solution to Jiles Law of Approach [2] was also successfully found to accurately model the evolution of external $\mathbf{B}$ with stress, in the case of a vertical inductor field [1]. This dual approach has been recently generalized to any field direction, when stress and field are no more parallel [3].

Our goal is now to characterize the magnetization $\mathbf{M}$ inside the material while internal pressure is increasing. Since $\mathbf{M}$ cannot be directly measured with our geometry, our original approach is to solve an inverse problem: Locapi, an inverse algorithm developed in our laboratory [4], has been used to achieve this. In the paper, the experimental setup and the method of inversion are presented. Using results derived in [3], a direct vectorial model for the variations of magnetization with pressure is introduced. The magnetization resulting from this model is used to predict the magnetic induction signature which would

Manuscript received May 30, 2010; revised October 28, 2010; accepted November 29, 2010. Date of current version April 22, 2011. Corresponding author: A. Viana (e-mail: anviana@yahoo.com).

Color versions of one or more of the figures in this paper are available online at http://ieeexplore.ieee.org.

Digital Object Identifier 10.1109/TMAG.2010.2097242 be measured by a tri-axial sensor. Comparison with measurements we performed on 8 tri-axial magnetic sensors shows the validity of this model.

\section{EXPERIMENTAL CHARACTERIZATION OF MAGNETOELASTIC EFFECTS}

\section{A. Experimental Setup}

All measurements were conducted in the LMMCF (Laboratory of Magnetic Metrology in Weak Fields), located in Grenoble, France. In this experimental facility, research and measurements are conducted in the area of very low magnetic fields (around a few nT). A magnetic environment simulator has been designed and built to compensate local earth field and to create any field between $\pm 70 \mu \mathrm{T}$, with an homogeneity close to $10^{-3}$ over a large volume $(27 \times 2 \times 2 \mathrm{~m})$ (Fig. 1(d)) [5]. The system is based on a tri-axial set of coils, driven by bipolar generators. Mock-ups can be mounted on a trolley driven over a pair of rails by an electrical motor along the longitudinal direction, allowing magnetic signature measurements in a -1.5 to $1.5 \mathrm{~m}$ range above fixed magnetic sensors.

The prototype is a ferromagnetic hollow cylinder of axis $\mathrm{Z}$. Dimensions are length $\mathrm{L}=0.5 \mathrm{~m}$, internal radius $\mathrm{R} \gg \mathrm{e}$, e being the thickness of the cylinder. The latter, filled with hydraulic oil, is subjected to an internal pressure up to 100 bar, driven by an external pump (Fig. 1(c)). A pressure sensor mounted inside the cylinder allows an accurate measurement of internal pressure.

The system was designed in order to let both ends of the cylinder be free in displacement when pressure increases. Thus, the mechanical approach was to work in plane stresses. As a consequence, the $\sigma_{\mathrm{zz}}$ stress tensor component is null. For our mock-up, the main stress is $\sigma_{\theta \theta}$. In addition, stresses are proportional to internal pressure. Thus, plots representing magnetization versus stress are identical to those representing magnetization versus internal pressure (i.e., radial stress $\sigma_{\mathrm{RR}}$, or P).

For thin shells, magnetization remains necessarily tangential to the shell. This is the consequence of the form effect: a magnetization orthogonal to the surface cannot occur due to the demagnetizing field in that direction.

When the cylinder is subjected to a vertical inductor field, magnetization in the shell will be parallel to the main stress $\sigma_{\theta \theta}$ 


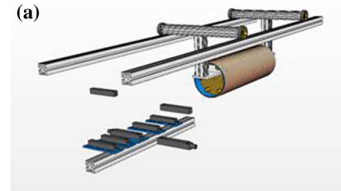

(b)
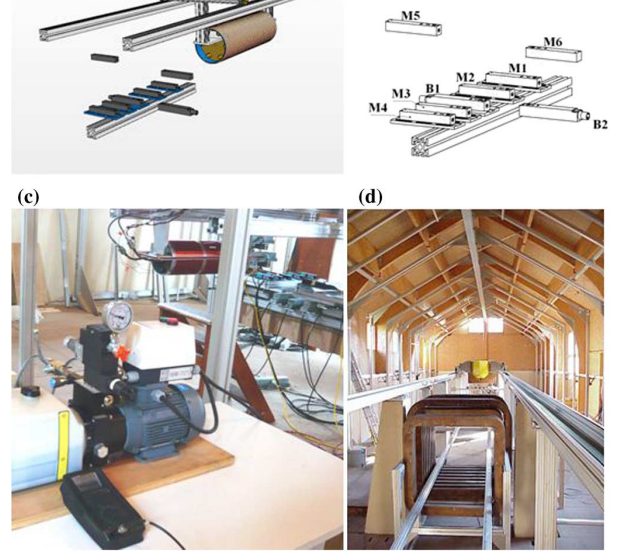

Fig. 1. (a), (b) Location of the sensors. (c) The hydraulic pump, and the cylinder inserted into the longitudinal solenoid. (d) The LMMCF metrology building is oriented in the N-S direction. The coils used for controlling the ambient field are visible, as well as the rails used for signature measurements.

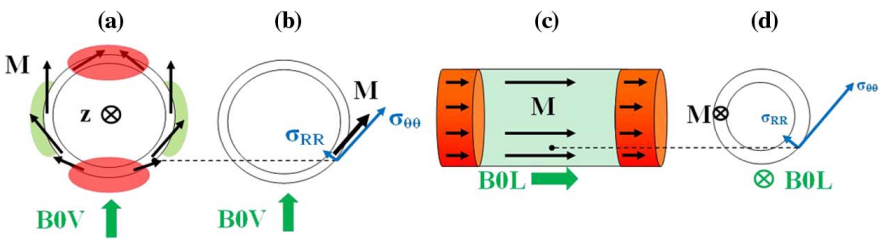

Fig. 2. Expected magnetization versus stress configuration for two different inductor fields B0V (vertical field) and B0L (longitudinal field). Dark (resp. light) zones exhibit a strong (resp. minimal) form effect. For thin shells, magnetization is necessarily tangential to the shell, i.e., parallel to $\sigma_{\theta \theta}$.

(Fig. 2(a) and (b)). Conversely, a longitudinal inductor field will lead to a configuration where stress $\sigma_{\theta \theta}$ and $\mathbf{M}$ are orthogonal (Fig. 2(c) and (d)).

The cylinder is inserted into a longitudinal solenoid (Fig. 1(b)) used for both demagnetization and polarization operations. Two Kepko power supplies allow the generation of a longitudinal field up to $9000 \mathrm{~A} / \mathrm{m}$. Eight tri-axes Fluxgate sensors are used (denoted M1 to M6, B1 and B2) with a dynamic range $\pm 100 \mu \mathrm{T}$, a bandwidth from $\mathrm{DC}$ to $1 \mathrm{KHz}$, a sensitivity of $1 \mathrm{nT}$. Fig. 1 shows the physical location of the eight sensors.

The set of eight sensors allows two kinds of measurements:

- Signature measurement (Fig. 3): in this case, the cylinder is moving along the rails and eight signatures are performed. Given the location of the sensors, five signatures are equivalent.

- Static measurement during an HPP cycle (Fig. 4): in this case, inductor field is set to $\mathbf{H}$. Then pressure is raised up to value $\mathrm{P}$, then released. The cylinder is static, centered above sensor B1. Variation of induction versus pressure is monitored on the eight sensors.

\section{B. HPP Cycles Measurements}

Measurements were firstly focused on the variation of external induction $\mathbf{B}$ [3], while pressure was raised, then relaxed, under a constant applied induction $\mathbf{B}_{\mathbf{0}}$ of any direction. Experimentally, such HPP cycles measurements consist of three steps. Firstly, a particular initial magnetic state is conferred to the cylinder: zero-stress demagnetization, or anhysteretic polarization under a given applied external induction $\mathbf{B}_{\mathbf{P O L}}$. Secondly, the inductor field is set to B0. Finally, pressure is raised up to $100 \mathrm{bar}$, and then relaxed to 0 bar. At a step of the HPP process,
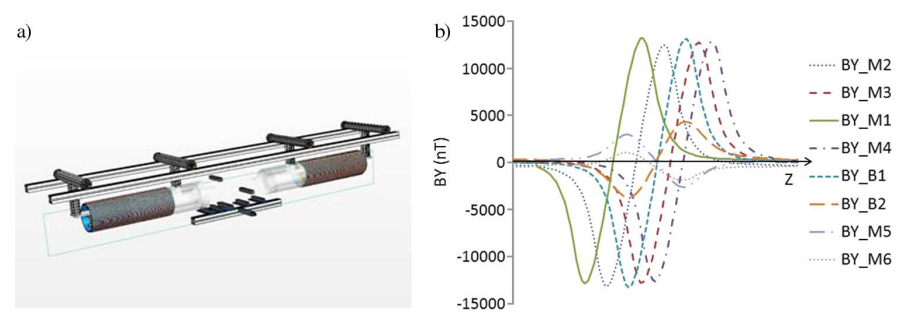

Fig. 3. Induction B signature. (a) The cylinder is translated over the eight sensors, producing the magnetic signature. (b) The typical magnetic signature curves obtained for each of the three components of induction B. The plot represents the vertical component BY of induction B under a longitudinal inductor field $\mathrm{B} 0 \mathrm{~L}=40 \mu \mathrm{T}$. The plot is characteristic of the orthogonal component $\mathrm{Y}$ of a $\mathrm{Z}$ oriented dipole signature. a)
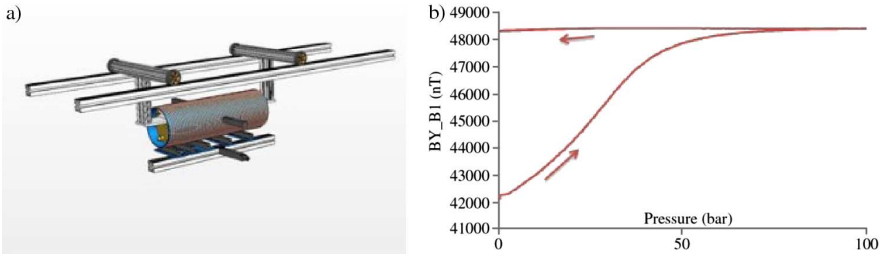

Fig. 4. HPP cycle: external induction change measurement under a constant vertical inductor field B0V, while pressure is first raised, then released. (a) The cylinder is static, centered above B1 sensor. (b) Vertical component BY_B1 of induction measured on centered sensor B1, during a HPP cycle under a vertical inductor field $\mathrm{B} 0 \mathrm{~V}=40 \mu \mathrm{T}$.

a magnetic signature can be performed under a given static pressure. In this case, the cylinder, initially static (Fig. 4(a)), is translated along the sensors (Fig. 3(a)) while pressure $\mathrm{P}_{\mathrm{MES}}$ is maintained. Once the signature is performed, the cylinder is sent back to its original location, and the end of the HPP cycle (pressure release for example) is recorded. This particular magnetic signature, achieved under pressure, is a reliable way to validate any modeled magnetization distribution, by comparison with its predicted signature.

Several HPP cycles series were done with different initial magnetic states and inductor fields (Fig. 5). In all cases, external induction converges towards its anhysteretic value under pressure, independently from the initial polarization configuration. In addition, measurements showed that the measured induction during an HPP cycle was linear with regards to the inductor field. Consequently, induction changes observed on each sensor during an HPP cycle performed under any inductor field $\mathbf{B 0}=\lambda \mathbf{B 0 L}+\mu \mathbf{B 0 V}$ can be deduced from those measured separately using 2 HPP cycles, operated under the two reference inductor fields BOL then $\mathbf{B O V}$ :

$$
\boldsymbol{B}(P, \boldsymbol{B 0})=\lambda \boldsymbol{B}(P, \boldsymbol{B O} \boldsymbol{L})+\mu \boldsymbol{B}(P, \boldsymbol{B O} \boldsymbol{V})
$$

The experienced linearity of $\mathbf{B}$ is in agreement with the expected linear behavior of induction in the Rayleigh low field region. These first results found for external $\mathbf{B}$ were then used to build our vectorial model for intrinsic magnetization variation due to magnetostriction.

\section{MAGNetiZATION Model}

\section{A. Locapi, an Algorithmic Inversion Software}

Locapi is an inverse magnetostatic algorithm [4] based on the method of moments, and dedicated to thin shells. For such geometries, the magnetization is assumed to remain tangential to the shell, given the unfavorable form effect preventing the magnetization to have a component perpendicular to the surface. 


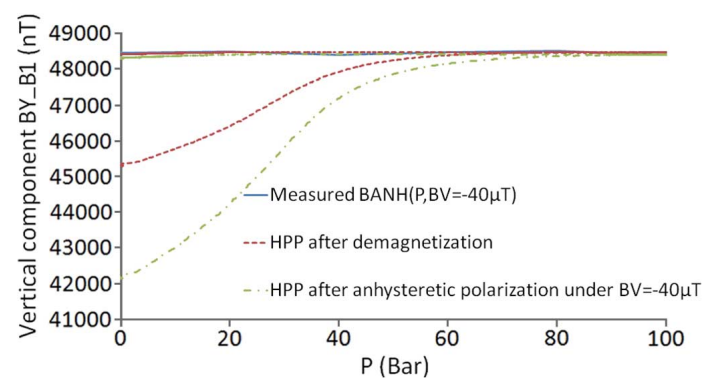

Fig. 5. Variation of the vertical component BY_B1 of induction B on sensor B1 with pressure $\mathrm{P}$ under inductor field $\mathrm{B} 0 \mathrm{~V}=40 \mu \mathrm{T}$. Induction tends towards its anhysteretic value independently from the initial magnetic state of the cylinder.

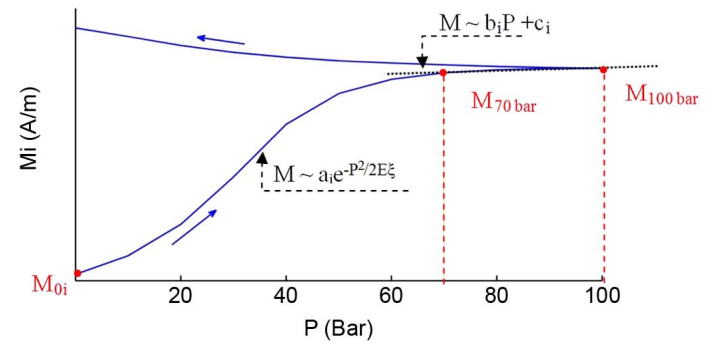

Fig. 6. Typical variation of any component Mi of magnetization Min the shell, determined from measurements by using Locapi.

In addition, magnetization is considered as constant through the cylinder shell thickness. Based on induction $\mathbf{B}$ signatures (Fig. 3(b)) measured by external magnetic sensors located in the vicinity of a meshed shell, Locapi computes the distribution of magnetization $\mathbf{M}\left(\boldsymbol{\Omega}_{\mathbf{i}}, \mathbf{B} \mathbf{0}\right)$ at element $\Omega_{\mathbf{i}}$, inside the cylinder, for a given inductor field $\mathbf{B 0}$.

In this study, Locapi was used to reconstruct magnetization variations in the cylinder using measurements from HPP cycles. To achieve this, the following approach was used: inductions

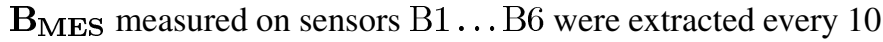
bar during HPP cycles, and used to calculate the magnetization predicted by Locapi at each pressure step. Sensors M5 and M6 data were not used for inversion, but dedicated to the validation of the inversion result: the magnetization reconstructed by Locapi at pressure $\mathrm{P}$ was used as the magnetic source term in the direct problem solver feature of Locapi, yielding the predicted induction signature $\mathbf{B}_{\text {PRED }}$ on the M5 and M6 sensors. $\mathbf{B}_{\text {PRED }}$ was then compared with $\mathbf{B}_{\text {MEs }}$ measured on sensors M5 and M6.

This protocol gave the measured vectorial laws $\mathbf{M}\left(\Omega_{\mathrm{i}}, \mathrm{P}, \mathbf{B 0 L}\right)$ and $\mathrm{M}\left(\Omega_{\mathrm{i}}, \mathrm{P}, \mathbf{B O V}\right)$, for $\mathrm{P}$ in $\{0 ; 10 ; \ldots ; 100\}$ bar. All Locapi inversions showed that magnetization, for each element of the cylinder, follows firstly an exponential law, then a linear law with stress (Fig. 6). It is worth noting that a similar behavior was already noticed for the variation of external induction $\mathbf{B}$ with pressure [3].

\section{B. An Analytical Model for Magnetization Changes}

In [3], an analytical vectorial law for the variation of induction $\mathbf{B}$ with stress was derived for any induction B0. This approach to the problem led to 3 analytical solutions for each component $\mathrm{B}_{\mathrm{j}}$ of $\mathbf{B}(\mathrm{j}=\mathrm{X}, \mathrm{Y}$ or $\mathrm{Z})$ :

$$
B_{j}(P, \boldsymbol{B 0})=a_{j}(B 0) e^{-\frac{P^{2}}{2 E \xi}}+b_{j}(B 0) \cdot P+c_{j}(B 0)
$$

$\mathrm{E}(\mathrm{Pa})$ is the Young's modulus of the material. $\xi\left(\mathrm{J} / \mathrm{m}^{3}\right)$ is an intrinsic parameter of the material, accounting for the rate of change of induction with mechanical stress [2]. $\xi$ was determined using a fitting procedure. For $\mathrm{P}$ expressed in bar and $\mathrm{E}=2.05 .10^{11} \mathrm{~Pa}, \xi \approx 2.5 \times 10^{-9} \mathrm{~J} / \mathrm{m}^{3}$. This intrinsic parameter of the material was found to be equal for the three laws $B_{j}$, accounting for an isotropic magnetoelastic behavior of the material at the scale of the prototype.

The approach used for $\mathbf{M}$ was to extend the law found in [3] for $\mathbf{B}(\mathrm{P}, \mathrm{B0})$. The analytical vectorial law describing the variations of magnetization $\mathbf{M}\left(\Omega_{\mathrm{i}}, \mathrm{P}, \mathbf{B}\right)$, for any point $\Omega_{\mathrm{i}}$ inside the shell, is expected to be similar to (2). The reason for that lies in [4]: for thin ferromagnetic shells, the law of external induction and intrinsic magnetization are similar. Inversions performed with longitudinal and vertical inductor fields confirm this theoretical result.

Given the physical symmetries of the problem, for a vertical inductor field, magnetization $\mathbf{M}$ in the shell will have a null longitudinal Z component. Conversely, under a longitudinal inductor field, magnetization will have a single longitudinal component:

$$
\begin{aligned}
\boldsymbol{M}\left(\Omega_{i}, P, \boldsymbol{B O V}\right)= & M_{X}\left(\Omega_{i}, P, \boldsymbol{B O} \boldsymbol{V}\right) \boldsymbol{X} \\
& +M_{Y}\left(\Omega_{i}, P, \boldsymbol{B O} \boldsymbol{V}\right) \boldsymbol{Y} \\
\boldsymbol{M}\left(\Omega_{i}, P, \boldsymbol{B O} \boldsymbol{L}\right)= & M_{Z}\left(\Omega_{i}, P, \boldsymbol{B O} \boldsymbol{L}\right) \boldsymbol{Z}
\end{aligned}
$$

The magnetization law for any inductor field can be reconstructed using (1), by linear combination of (3) and (4). In addition, $\xi$ being an intrinsic parameter of the material, its fitted value found in [3] was reused for the law in magnetization. Hence, the analytical law for the transverse component $\mathrm{M}_{\mathrm{X}}$ of magnetization $\mathbf{M}$ should be expressible as

$$
\begin{aligned}
M_{X}\left(\Omega_{i}, P, \boldsymbol{B O} \boldsymbol{V}\right)= & a_{X}\left(\Omega_{i}, \boldsymbol{B} \mathbf{O}\right) e^{-P^{2} / 2 E \xi} \\
& +b_{X}\left(\Omega_{i}, \boldsymbol{B} \mathbf{0 V}\right) P+c_{X}\left(\Omega_{i}, \mathbf{B} \mathbf{V}\right)
\end{aligned}
$$

Similar laws for $\mathrm{M}_{\mathrm{Y}}$ and $\mathrm{M}_{\mathrm{Z}}$ are expected. Thus, the determination of the vectorial law $\mathrm{M}\left(\Omega_{\mathrm{i}}, \mathrm{P}, \mathbf{B} \mathbf{0}\right)$ requires knowledge of nine unknown coefficients for each element of the meshed cylinder. A first method for determining coefficients $a, b$, and c is to use a fitting algorithm, using (5) as a target function. This method was successfully tested.

Nevertheless, a more physical and straight approach can be used. Equation (5) can be decomposed in two terms: an exponential and a linear term.

For $\mathrm{P}<70$ bar, the behavior of magnetization can be approached by

$$
M_{J}\left(\Omega_{i}, P, B \mathbf{B}\right)=a_{i J}(\mathbf{B O}) e^{-P^{2} 2 E \xi}
$$

Equation (6) is the only analytical solution to the Jiles-Atherton equation [1]. This particular solution is only applicable for the case of a material whose anhysteretic induction is independent from stress.

For $\mathrm{P}>70 \mathrm{~B}$, while $\mathrm{P}$ is increasing, the exponential term becomes negligible compared to the linear term, given the value found for $\xi$ in [1]. The linear term accounts for the variations of induction under high level of pressure. In this case, magnetization is close to the anhysteretic curve. We already mentioned that the latter is either a constant (vertical polarization field), or a linear function of pressure (longitudinal polarization field). Consequently, under high levels of pressure, the law describing magnetization changes must follow the same behavior as the anhysteretic magnetization. 


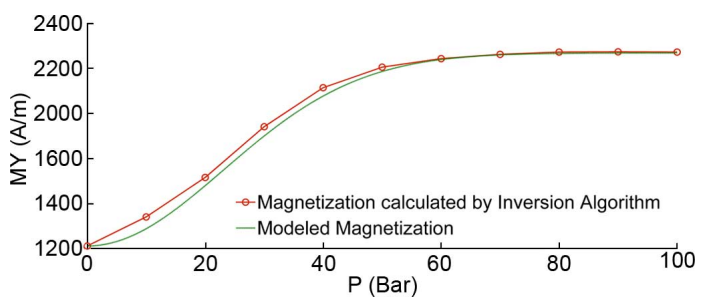

Fig. 7. Modeled variation of vertical component of magnetization with pressure on an element of the meshing using our direct model (-). Model shows good agreement with the variation of magnetization calculated using Locapi (o).

Given this, determination of the coefficients can be experimentally done: $\mathrm{P}=0$ bar $(\mathbf{M}=\mathbf{M 0})$ in (5) yields

$$
a_{X, Y, Z}\left(\Omega_{i}\right)=M 0_{X, Y, Z}\left(\Omega_{i}\right)-c_{X, Y, Z}\left(\Omega_{i}\right)
$$

Similarly, with $\mathrm{P}=70$ bar $(\mathbf{M}=\mathbf{M 7 0})$ and $\mathrm{P}=$ 100 bar $(\mathbf{M}=\mathbf{M 1 0 0})$ in (5):

$$
\begin{aligned}
b_{X, Y, Z}\left(\Omega_{i}\right) & =\frac{M 100_{X, Y, Z}\left(\Omega_{i}\right)-M 70_{X, Y, Z}\left(\Omega_{i}\right)}{100-70} \\
c_{X, Y, Z}\left(\Omega_{i}\right) & =\frac{100 M 70_{X, Y, Z}-70 M 100_{X, Y, Z}}{100-70}
\end{aligned}
$$

This method requires six inversions to fully determine (5):

- Three inversions performed at $\mathrm{P}=0$ bar, $\mathrm{P}=70$ bar, and $\mathrm{P}=100$ bar, under a longitudinal inductor field $\mathbf{B O L}$, yield values for $\mathrm{a}_{\mathrm{Z}}\left(\Omega_{\mathrm{i}}\right), \mathrm{b}_{\mathrm{Z}}\left(\Omega_{\mathrm{i}}\right)$ and $\mathrm{c}_{\mathrm{Z}}\left(\Omega_{\mathrm{i}}\right)$ for all elements $\Omega_{\mathrm{i}}$ of the meshing.

- The three subsequent inversions, at same pressures, but under a vertical inductor field, yield values for $\mathrm{ax}\left(\Omega_{\mathrm{i}}\right)$, $\mathrm{b}_{\mathrm{X}}\left(\Omega_{\mathrm{i}}\right), \mathrm{c}_{\mathrm{X}}\left(\Omega_{\mathrm{i}}\right), \mathrm{ar}_{\mathrm{Y}}\left(\Omega_{\mathrm{i}}\right)$, by $\left(\Omega_{\mathrm{i}}\right)$ and $\mathrm{c}_{\mathrm{Y}}\left(\Omega_{\mathrm{i}}\right)$.

Finally, by linear combination of the scalar laws for longitudinal and vertical inductor fields, a direct vectorial model for the variation of magnetization under any inductor field was obtained, and validated with the experimental HPP cycles.

Fig. 7 shows the validity of the proposed model, by comparing it to measurements obtained from inversion for one element of the meshing.

\section{Experimental Validation}

The cylinder was meshed in 320 elements. Determination of coefficients $\mathrm{aX}_{X, Y, Z}, \mathrm{~b}_{\mathrm{X}, Y, Z}, \mathrm{C}_{X, Y, Z}$ was achieved using reference inductor fields $\mathbf{B 0 L}=40 \mu \mathrm{T}$ and $\mathbf{B O V}=80 \mu \mathrm{T}$.

Several HPP cycles were performed under various inductor fields in any direction. During each HPP cycle, pressure was maintained at different values, and signature measurements were performed. The predicted signatures of the direct model were then compared to the measured signatures (Fig. 8).

Note that coefficients $\mathrm{ax}, \mathrm{Y}, \mathrm{Z}\left(\Omega_{\mathrm{i}}\right)$ depend on the initial magnetic state, and thus, require to be recalculated for each HPP cycle initiated with a non-demagnetized state. Validation was also successfully achieved using different anhysteretic magnetizations as initial magnetization states.

The predicted signatures were in good agreement with the measured ones, for any inductor field, and different magnetic initial states. Fig. 8 shows the predicted magnetization at 50 bar, under an inductor field of longitudinal and vertical components
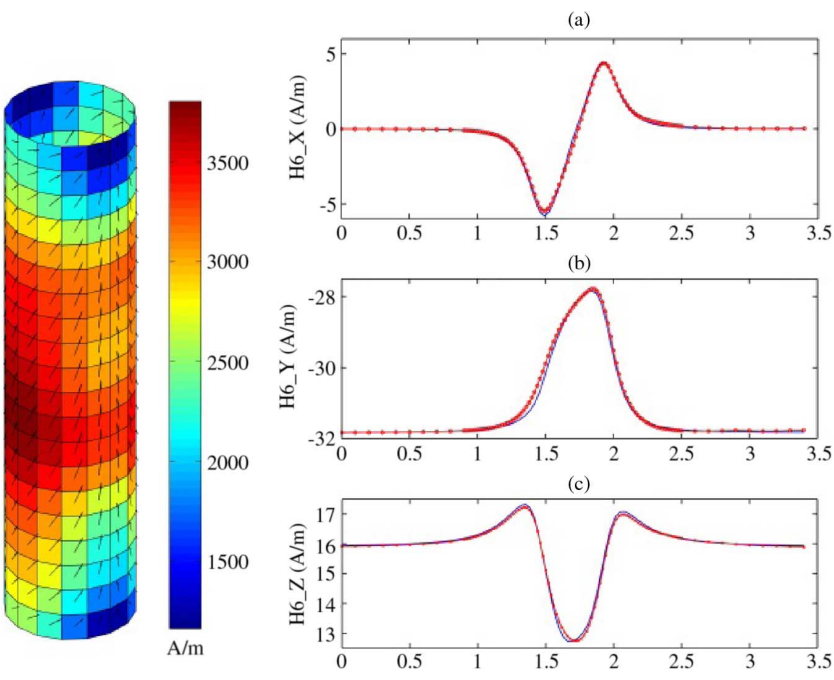

Fig. 8. (Left) Predicted magnetization in the shell, at $\mathrm{P}=50$ bar, under inductor $\mathrm{B}=\mathrm{BL}+\mathrm{BV}, \mathrm{BL}=20 \mu \mathrm{T}, \mathrm{BV}=40 \mu \mathrm{T}$. (Right) Predicted versus measured magnetic signatures on sensor M6 for the three components of external magnetic field.

$-20 \mu \mathrm{T}$ and $+40 \mu \mathrm{T}$. Comparison between the predicted and the measured signatures show a relative error less than $8 \%$.

\section{CONCLUSION}

In this paper, an inverse problem algorithm made it possible to characterize magnetization variations due to magnetostriction inside a hollow cylinder exhibiting non negligible demagnetizing field. An analytical expression was introduced to model these magnetization variations. As expected, this law is closely linked to the law followed by another important magnetic feature, the external induction $\mathbf{B}$ measured outside the cylinder. Thus, we showed that the main conclusions, already found for external induction $\mathbf{B}[3]$ were still valid for $\mathbf{M}: \mathbf{M}$ tends towards the anhysteretic value, its variations with pressure are proportional to the inductor field strength and decoupled by axes. As a consequence, a vectorial magnetostriction model is proposed. Its validity is proven by predicting the external field with an error less than $8 \%$ with respect to measurements, for any direction of the inductor field.

\section{REFERENCES}

[1] A. Viana, L.-L. Rouve, G. Cauffet, and J.-L. Coulomb, "Magneto-mechanical effects under low fields and high stresses-Application to a ferromagnetic cylinder under pressure in a vertical field," IEEE Trans. Magn., vol. 46, no. 8, pp. 2872-2875, 2010.

[2] D. C. Jiles, "Theory of the magnetomechanical effect," J. Phys. D: Appl. Phys., vol. 28, pp. 1537-1546, 1995.

[3] A. Viana, L.-L. Rouve, G. Cauffet, and J.-L. Coulomb, "Analytical model for external induction variations of a ferromagnetic cylinder undergoing high mechanical stresses in a low magnetic field of any orientation," IEEE Trans. Magn., vol. 47, no. 5, May 2011

[4] O. Chadebec, J.-L. Coulomb, G. Cauffet, and J.-P. Bongiraud, "How to well pose a magnetization identification problem," IEEE Trans. Magn., vol. 39, no. 3, pp. 1634-1637, 2003.

[5] J.-P. Bongiraud, G. Cauffet, C. Jeandey, and P. Le Thiec, "An optimization method for magnetic field generator," in Marelec 1999, Conf. Proc., pp. 161-168. 